\title{
Implantation of Pacemaker in a Patient with Dextrocardia, Persistent Left Superior Vena Cava, TGA and Heart Block
}

\section{Murat KERKÜTLÜOĞLU* and Mahmut TUna KATIRCIBAŞI}

Cardiology Department, Kahramanmaraş Sütçüimam University, Turkey

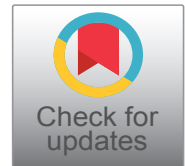

*Corresponding author: Murat KERKÜTLÜOĞLU, Cardiology Department, Kahramanmaraş Sütçüimam University, Turkey

\section{Abstract}

Congenital anomalies of the heart may generate difficulties for cardiac invasive procedures. Dextrocardia is a rare congenital heart disease in which the apex of the heart is located on the right side of the chest. Patients with transposition of great arteries (TGA) abnormalities are at risk for complete cardiac block and usually require a permanent cardiac pacemaker in long-term follow-up. The persistent left superior vena cava (PLSCV) is a rare venous anomaly, often associated with abnormalities of the cardiac transduction system. It is usually seen by chance during permanent pacemaker implantation. Even if electrodes are not impossible to be placed through this abnormal venous structure, it can be challenging. In this study, we present a permanent pacemaker implantation to a 31-year-old female patient with a combination of dextrocardia, PLSVC, and TGA due to complete heart block.

Congenital anomalies of the heart can make transvenous procedures such as right heart catheterization or pacemaker implantation difficult. During embryological development, PLSVC occurs as a result of obliteration of the proximal part of the right anterior and right cardinal veins [1]. The left anterior cardinal vein creates a vena cava superior that opens to the right atrium through the left coronary sinus or directly to the left atrium. The persistent left superior vena cava (PLSCV) is rare but the most common systemic venous anomaly, which is $0.5 \%$ in general population and $3-10 \%$ in congenital heart defects [2]. Another anomaly is congenital dextrocardia, which is a rare congenital heart defect in which the heart is predominantly localized on the right side of the thorax. The reported dextrocardia frequency is 1 in 10,000 live births [3]. It is responsible for $0.5 \%$ of cases of adult congenital heart disease [4]. Transposition of great arteries (TGA) is a rare congenital anomaly that represents about $0.5 \%$ of congenital heart disease. The oxygenated blood in the pulmonary veins flows from the left atrium to the right ventricle which is compatible with the tricuspid valve and is pumped into the incompatible aorta [5]. In patients with TGA, the AV node and His bundle have an unusual position, resulting in dysfunction of the impulse system. Approximately
\end{abstract}

$2 \%$ of patients with TGA develop complete AV block per year [6]. Our patient whom TGA and dextrocardia were combined with a PLSVC had previously developed a full AV block, and repeated attempts of pacemaker implantation with a subclavian approach had failed, and epicardial pacemaker had been inserted. The VVI-R pacemaker was implanted successfully with a subclavian approach to the patient who applied to us due to the end of the battery life.

\section{Case}

A 31-year-old female patient was admitted to our clinic for controls of the implanted pacemaker due to complete heart block, and after the evaluation of the battery life, battery replacement was planned and the patient was hospitalized. The patient's history was learned to have no surgical story L-TGA, dextrocardia and PLSVC diagnoses (Figure 1). It was learned that the epicardial pacemaker was applied because of failed attempts of four times to attach the endocardial VVI-R pacemaker, two of which are 20 years ago and another two are 12 years ago. The blood pressure of the patient whose ECG was in pacemaker rhythm was measured at $125 / 80 \mathrm{mmHg}$ and heart rate at $60 / \mathrm{min}$. The patient was evaluated with preoperative computed tomography (CT) due to present congenital anomalies, superior vena cava was in left and arcus aorta in right. The TGA-compatible imagination, catheter imagination in vena cava superior, and mirror imagination in solid organs of the abdomen in accordance with situs inversus was observed. The patient who was taken to the laboratory for pacemaker replacement was given venography from the right and left tibial veins. Then, right ventricular anatomy was evaluated using the main femoral vein.

Right ventricle (RV) was cannulated by making punc- 


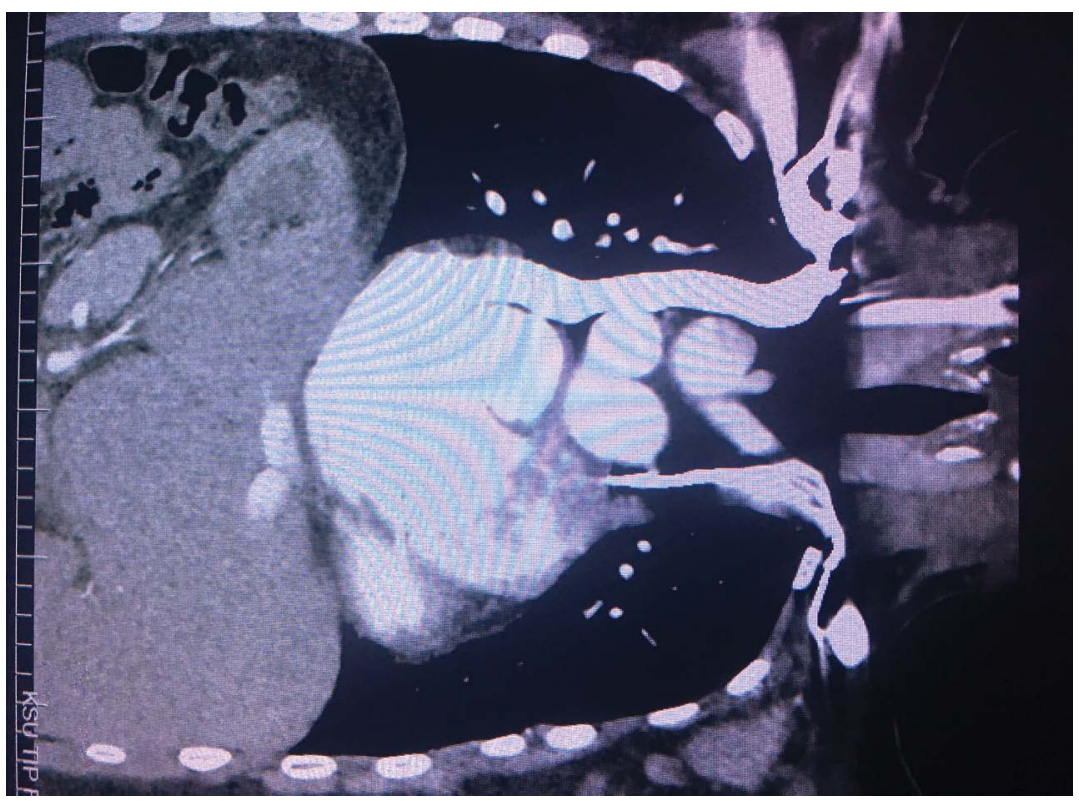

Figure 1: Thorax ct angiography.

ture from the right subclavian venous tract and referencing the catheter sent by the right ventricle femoral route. However, a gap was created in the left subclavian region because the lead could not grow. The right ventricle was cannulated with hydrophilic wire and MP catheter. Subsequently, a 0.32-inch Amplatz wire was sent to increase the support force by pulling the hydrophilic wire while the MP catheter was in the RV. The rupturable sheet used for cardiac resynchronization pacemakers implantation was entered into RV via this wire. During this process, very slow and gentle maneuvers were applied to avoid possible RV rupture. Then active VVI-R pacemaker lead was placed into the right ventricle through this system. After placement, sheat was pulled under the control of fluoroscopy by applying the rupture and pull procedure performed just as in CRT lead placement (cardiac resynchronization therapy). Measurements were recorded and a generator was installed and properly closed. In the same session, the epicardial pace generator was removed and the process finished.

\section{Discussion}

Most TGA cases are together with other cardiac anomalies such as ventricular septal defect, pulmonary stenosis, Ebstein-like malformation, and these related anomalies determine the symptoms of congenital anomaly [7]. However, less than $20 \%$ of TGA patients with no other cardiac abnormalities are asymptomatic and symptoms of arrhythmia and heart failure occur later in life [8]. In such cases, the morphological right ventricle (RV) as a systemic pump may gradually become dysfunctional, and concomitant tricuspid valve insufficiency may occur due to the excessive volume loading of the morphological RV. Natural History of uncomplicated TGA patients shows more frequent progressive AV transmission loss than normal population [7]. A full
AV block is often caused by the vulnerability of His bundles to fibrosis [9].

Pacemaker lead placement can be challenging in patients after Mustard and Senning operations for transposition of the great arteries (D-TGA). Chakrabarti and his colleagues performed cardiac pace implantation with RF catheter in D-TGA patients in which they mentioned the difficulties of pacemaker implantation [10]. In our case, we had to use coronary sinus delivery system for lead implantation. There are difficulties of lead implantation in both types of implantation. This difficulty can be overcome using techniques other than the standard techniques used in the lead impalntation.

Dextrocardia is clinically characterized by three subgroups based on the position of heart chambers and abdominal organs. First, the heart is on the right side of the chest and the apex part points to the right portion of the body. Extractions usually bring along other intracardiac anomalies and are usually caused by a positional shift and rotation of the right heart. Second, only the heart is in the opposite position, but other internal organs are positioned appropriately. The third is dextrocardia (situs inversus or mirror image dextrocardia); anterior-posterior relationships of different parts of the heart are normal, but right-to-left orientation is reversed [11]. The patient was diagnosed with a mirror image dextrocardia.

The persistent left superior vena cava (PLSVC) is a rare congenital anomaly and can be seen in conjunction with other cardiac or vascular malformations [12]. According to venous drainage locations, PLSVC can be divided into 4 types: Type I PLSVC forms drainage to the right atrium via the coronary sinus; type II PLSVC is connected to the right atrium via the coronary sinus but has a connection with the left atrium, so the right-toleft shunt occurs; type III PLSVC makes shunt right to 
left from the left atrium; type IV PLSVC is directly connected to the left pulmonary vein [13]. Type I accounts for about $90 \%$ of all PLSVC cases [12]. Although Type I PLSVC generally does not have a significant hemodynamic outcome, it provides a large amount of current to the atrium through the coronary sinus, and may especially expand the sinus ostium in the absence of the right vena cava superior. Since sinoatrial node, atrioventricular node and His bundles are structurally close to the coronary sinus, the incidence of arrhythmias in PLSVC patients is also higher. The patient reported here had type I PLSVC with AV complete block.

Due to abnormal anatomy, lead placement to the right ventricular through PLSVC and coronary sinus can be technically difficult. Serious complications such as arrhythmia, cardiogenic shock, and even cardiac arrest have been reported when PLSVC has been manipulated by a guide wire or catheter [14]. The use of advanced lead transmission systems may be needed to reach the right ventricle.

Our case was a one that had previously undergone repeated subclavian unsuccessful pacemaker implantation. She had to come to the electrophysiology lab every three years for a battery replacement due to the epicardial pacemaker. The battery exchange process we implemented was the $8^{\text {th }}$ for the patient. The right ventricular angiography via femoral vein, pulmonary CT angiography, MP catheter, Amplatz wire and CRT transfer system were used to attach a permanent pacemaker through the subclavian vein.

After a thorough examination and understanding the vascular anatomy, abnormalities of the veins and heart chambers, we report a rare case successfully treated with pacemaker implantation in a patient with $\mathrm{AV}$ complete block associated with TGA, dextrocardia, and PLSVC congenital anomaly. It should be noted that there are no standard criteria to be followed in the treatment of patients with congenital abnormalities.

\section{References}

1. Edwards J, DuShane J (1950) Thoracic venous anomalies. Arch Pathol 49: 514-537.
2. Petronzelli S, Patruno N, Pontillo D (2008) Persistent left superior vena cava: Diagnosis with saline contrast echocardiography. Heart 94: 835.

3. Elbasheer E, Habib A, Salam A (2010) Primary angioplasty and later elective multivessel stenting in a patient with dextrocardia: A case report and literature review. J Invasive Cardiol 22: 125-128.

4. Offen S, Jackson D, Canniffe C, Choudhary P, Celermajer DS (2016) Dextrocardia in Adults with Congenital Heart Disease. Heart Lung Circ 25: 352-357.

5. Warnes CA (2006) Transposition of the great arteries. Circulation 114: 2699-2709.

6. Anderson RH, Becker AE, Arnold R, Wilkinson JL (1974) The conducting tissues in congenitally corrected transposition. Circulation 50: 911-923.

7. Graham TP Jr, Bernard YD, Mellen BG, Celermajer D, Baumgartner $\mathrm{H}$, et al. (2000) Long-term outcome in congenitally corrected transposition of the great arteries: A multi-institutional study. J Am Coll Cardiol 36: 255-261.

8. Beauchesne LM, Warnes CA, Connolly HM, Ammash NM, Tajik AJ, et al. (2002) Outcome of the unoperated adult who presents with congenitally corrected transposition of the great arteries. J Am Coll Cardiol 40: 285-290.

9. Prieto LR, Hordof AJ, Secic M, Rosenbaum MS, Gersony WM (1998) Progressive tricuspid valve disease in patients with congenitally corrected transposition of the great arteries. Circulation 98: 997-1005.

10. Chakrabarti S, Szantho G, Turner MS, Stuart G, Martin RP (2009) Use of radiofrequency perforation for lead placement in biventricular or conventional endocardial pacing after mustard or senning operations for D-transposition of the great arteries. Pacing Clin Electrophysiol 32: 1123-1129.

11. Rigatelli G (2007) Congenitally persistent left superior vena cava: A possible unpleasant problem during invasive procedures. J Cardiovasc Med (Hagerstown) 8: 483-487.

12. Povoski SP, Khabiri H (2011) Persistent left superior vena cava: Review of the literature, clinical implications, and relevance of alterations in thoracic central venous anatomy as pertaining to the general principles of central venous access device placement and venography in cancer patients. World J Surg Oncol 9: 173.

13. Pai RK, Cadman CS (2005) Persistence with a persistent left and absent right superior vena cava. Cardiol Rev 13: 163-164.

14. Grant RP (1958) The syndrome of dextroversion of the heart. Circulation 18: 25-36. 